\title{
Postoperative Early and Late Outcomes of Simultaneous Ureter and/or Bladder Resections and Reconstructions in Colorectal Malignancies with Locally Advanced Disease or Peritoneal Metastases
}

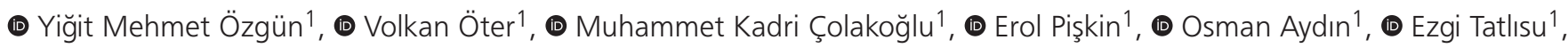 \\ (1) Cavit Ceylan², (1) Yusuf Bayram Özoğul³, (1) Erdal Birol Bostancı ${ }^{1}$ \\ 1 University of Health Sciences Turkey, Ankara City Hospital, Clinic of Gastroenterological Surgery, Ankara, Turkey \\ 2 University of Health Sciences Turkey, Ankara City Hospital, Clinic of Urology, Ankara, Turkey
}

\begin{abstract}
Objective: This study aimed to investigate the early and late outcomes of simultaneous urological procedures performed in patients who underwent surgery for primary or recurrent colorectal cancers with locally advanced disease or peritoneal metastases and effects of resection and reconstruction of the ureter and bladder on survival.

Materials and Methods: All patients with locally advanced disease or peritoneal metastases requiring concurrent urological procedures in our clinic between January 2014 and December 2020 were evaluated for this study. Only patients with bladder and ureter intervention were included in the study. Postoperative complications and urological complications were evaluated and classified according to the Clavien-Dindo classification. Imaging studies, interventional procedures, and follow-ups of patients with problems related to the urinary system in the long-term were recorded. The survival times of the patients were investigated.

Results: A total of 52 patients underwent simultaneous urological resection (ureter and bladder). As a synchronous urological procedure, an end-to-end anastomosis was performed after ureter resection in 12 patients, transureter anastomosis to 4, partial cystectomy in 20, ileal conduit with total cystectomy in 7, orthotropic neobladder in 1, and ureteroneocystostomy in 8 . The most common early complication in all patients was urinary leakage (10 patients), followed by wound infection (6 patients). The shortest and longest follow-up period of the whole group was 8 and 78 months, respectively, and the mean survival time was 38 months. No difference was found between patients with malignant ureter and benign ureter resections in terms of survival $(p=0.888)$.

Conclusion: In patients with clinical T4b and colorectal malignancies, en bloc resection should be the oncological procedure for bladder resections; if possible, organ-preserving surgery should be performed with sufficient negative margin. However, it is thought that the late outcomes of ureteroneocystostomy in ureter reconstruction are better and those of total cystectomy procedure are worse.
\end{abstract}

Keywords: Ureter, bladder, resection, colorectal malignancies, peritoneal metastases

\section{Introduction}

At the time of diagnosis, $5-10 \%$ of colorectal cancers are tumors with adjacent organ invasion (clinical T4b), and these tumors require multivisceral organ resections. To prevent the spread of the tumor and to provide R0 resection, an en bloc resection should be performed with the adjacent organ. These R0 resections increase the 5-year survival and decrease local recurrence rate $(1,2)$. The 5 -year survival rate of 50 -
90\% can be attained in patients in whom tumor-negative margin can be achieved with en bloc resection $(3,4)$. However, these multivisceral surgeries undoubtedly increase the risk of complications, and the morbidity and mortality rates after these surgeries vary between $7-76 \%$ and $0-10 \%$, respectively (4).

Colorectal tumors may be in close relationship with urological organs due to the anatomical location of the organs, and adhesions to these urological organs can also be malignant or benign. Therefore, resection of urological organs may be

Cite this article as: Özgün YM, Öter V, Çolakoğlu MK, Pişkin E, Aydın O, Tatlısu E, Ceylan C, Özoğul YB, Bostancı EB. Postoperative Early and Late Outcomes of Simultaneous Ureter and/or Bladder Resections and Reconstructions in Colorectal Malignancies with Locally Advanced Disease or Peritoneal Metastases. Bull Urooncol 2021;20(3):179-185

Address for Correspondence: Volkan Öter, University of Health Sciences Turkey, Ankara City Hospital, Clinic of Gastroenterological Surgery, Ankara, Turkey Phone: +90 5053366333 E-mail: otervolkan@gmail.com ORCID-ID: orcid.org/0000-0002-0639-1917 Received: 06.04.2021 Accepted: 06.05.2021 
required during colorectal surgeries. While total resection of affected organs may be needed, partial resections can be also performed. In cases with concurrence of colorectal malignancies, choosing between partial and total cystectomy in terms of oncological and functional outcomes may be difficult (5). Urological involvement can be seen in $7-20 \%$ of patients who undergo cytoreductive surgery and hyperthermic perfusion chemotherapy (CRS + HIPEC) due to peritoneal carcinomatosis, especially in those with metastases of colorectal malignancies, and simultaneous resection and reconstruction may be required $(6,7)$. After ureter resections, reconstruction procedures can be performed by various methods such as ureteroureterostomy, ureteroneocystostomy (UNS), transureteroureterostomy, and Boari flap reconstruction, and the effects of these methods on urinary leakage rate are uncertain (5).

In this study, we investigated the outcomes of simultaneous urological procedures performed in patients who underwent surgery for primary or recurrent colorectal cancers with locally advanced disease or peritoneal metastases. Moreover, we evaluated the early and late outcomes and effects of resection and reconstruction of the ureter and bladder on survival.

\section{Materials and Methods}

The study was planned as a retrospective analysis and before starting the study ethical permission was provided by the local hospital ethics committee. The ethical number of this study is E2-21-355.

All patients operated for colorectal cancer in our clinic between January-2014 and December-2020 were analyzed retrospectively. All patients with locally advanced disease or peritoneal metastases requiring concurrent urological procedures were evaluated. Only patients with bladder and ureter intervention were included in the study. Patients who received kidney, prostate, and urethra interventions were not included in the study because of differences in surgical techniques and complications. Both colorectal and urinary surgical margins were examined with peroperative frozen section in all patients. All patients who underwent surgery with curative intent and palliative resection were excluded from the study.

All patients were preoperatively examined for ureter and bladder invasion, hydronephrosis, and ureter dilatation from their radiological images. In a multidisciplinary council, patients who could undergo R0 resection received explanation of their radiological images before the treatment. Neoadjuvant chemoradiotherapy was performed to cases indicated for surgery due to rectal cancer and evaluated as locally advanced (T3, T4 and node positive).

Total colonoscopy was definitely performed in all patients preoperatively. Those who underwent cystoscopy, ureteroscopy, and prophylactic double-J catheter placement were recorded. All resections and reconstructions of the genitourinary tract and other resections performed in the same session were also recorded. Postoperative and urological complications were evaluated and classified according to the Clavien-Dindo classification (8). Pathological results of the resected primary tumor and urological specimen were examined separately. Imaging studies, interventional procedures, and follow-ups of patients with problems related to the urinary system in the longterm were recorded. The survival times of the patients were investigated.

Surgical Procedures: Laparotomy and resection were performed in all study patients. The colorectal cancer procedure was completed with relevant lymph node dissection according to the location of the tumor and en bloc resection of the adjacent organ. All patients underwent en bloc resection, regardless of whether the adhesion was malignant or a desmoplastic reaction. Patients with peritoneal metastases who could undergo complete cytoreduction (CCO) were included in the study. HIPEC was performed with the closed method in these patients. Catheters were placed after resection, and hyperthermic perfusion was implemented at $42{ }^{\circ} \mathrm{C}$ for $60 \mathrm{~min}$. Moreover, $200 \mathrm{mg} / \mathrm{m}^{2}$ oxaliplatin was used in all patients for the perfusion procedure. All urological and gastrointestinal anastomoses were performed after the HIPEC procedure.

Urological procedures: Unilateral or bilateral double-J stents were placed in some of the patients who had known T4 tumor and organ invasion in the same session before the operation. In some patients, stents could not be inserted because of severe stenosis. Reconstructive procedures for the ureter and bladder include uretero-ureterostomy, transuretero-ureterostomy, UNS, partial cystectomy, total cystectomy with orthotropic neobladder, and total cystectomy with ileal conduit.

\section{Statistic Analysis}

IBM SPSS Statistics program v. 21 (IBM Corp., Armonk, NY, USA) was used for data analysis. When evaluating the data, frequency distribution (numbers and percentages) was used for categorical variables and descriptive statistics (minimum, maximum, and median) was used for numerical variables depending on the results of the Kolmogorov-Smirnov test. Continuous variables were expressed as median (minimum-maximum) or mean \pm standard deviation where applicable. The Mann-Whitney $U$ test was performed for comparison of median values, and the mean differences were evaluated by Student's t-test. The chi-square test was performed to examine the relevance between two categorical variables. A p-value $<0.05$ was considered significant.

\section{Results}

Of the 657 patients who underwent surgery for colorectal malignancy in our clinic between January 2014 and December 2020, 52 underwent simultaneous urological resection (ureter and bladder). Moreover, 30 (57.7\%) patients were male and $22(42.3 \%)$ were female, and their mean age was $57.70 \pm 14.78$ years. CRS and HIPEC were performed in 21 (40.4\%) of these patients because of the presence of concurrent peritoneal metastases. While $24(46.2 \%)$ patients underwent primary malignancy surgery, 28 (53.8\%) patients were operated upon because of recurrence. Depending on the location, most tumors were located in the rectum, followed by the left colon and right colon (Table 1). The median hospital stay of the patients was 21 (7-103) days.

A total of 13 patients who underwent surgery for primary rectum carcinoma received long-term neoadjuvant chemoradiotherapy, 


\begin{tabular}{|c|c|}
\hline Total (n) & 52 \\
\hline Age $($ mean $\pm S D)$ & $57.705 \pm 14.784$ \\
\hline \multicolumn{2}{|l|}{ Gender } \\
\hline Male (\%) & $30(57.7 \%)$ \\
\hline Female (\%) & $22(42.3 \%)$ \\
\hline \multicolumn{2}{|l|}{ HIPEC } \\
\hline$+(\%)$ & $21(40.4 \%)$ \\
\hline - (\%) & $31(59.6 \%)$ \\
\hline $\begin{array}{l}\text { Lenght of stay (day) (median) } \\
\text { (range, min-max) }\end{array}$ & $\begin{array}{l}21 \\
(7-103)\end{array}$ \\
\hline Primary (\%) & $24(46.2 \%)$ \\
\hline Relapse (\%) & $28(53.8 \%)$ \\
\hline \multicolumn{2}{|l|}{ Primary tumor location } \\
\hline Rectum (\%) & $22(42.3 \%)$ \\
\hline Left colon (\%) & $19(36.5 \%)$ \\
\hline Right colon (\%) & $11(21.2 \%)$ \\
\hline \multicolumn{2}{|l|}{$\mathrm{N}$ stage of colorectal tumors } \\
\hline 0 & $38(73.1 \%)$ \\
\hline $1 a$ & $4(7.7 \%)$ \\
\hline $1 b$ & $5(9.6 \%)$ \\
\hline $2 a$ & $3(5.8 \%)$ \\
\hline $2 b$ & $2(3.8 \%)$ \\
\hline
\end{tabular}

and nine patients underwent surgery for recurrent rectum carcinoma. Left colon and rectal surgery was performed in 41 (78.8\%) patients, and right hemicolectomy was performed in 11 (21.2\%) patients. In these patients, bladder-related procedures were performed in 28 (53.9\%) patients, and ureter resection and reconstruction procedures were performed in 24 (46.1\%) patients. As a synchronous urological procedure, we performed an end-to-end anastomosis after ureter resection in 12 patients, transureter anastomosis in 4, partial cystectomy in 20, ileal conduit with total cystectomy in 7, orthotropic neobladder in 1 , and UNS in 8 (Table 2). In addition, bladder hitch procedure was performed to the psoas muscle in two patients who underwent UNS and thought to have a tight anastomosis.

In the pathological examination of the resection materials, the colorectal resection specimen of 49 patients revealed adenocarcinoma. While the result was benign in two patients who were thought to have recurrence preoperatively, the pathology of both colon and ureter resections was gastrointestinal stromal tumor (GIST) in one patient. The median number of harvested lymph nodes was $16.52 \pm 15.16$. Positive lymph node states ( $N$ stage) of the patients are presented in Table 1.

Operation time, postoperative complications, 30-day morbidity, 30-day mortality, survival, and long-term morbidity results are shown separately in Table 3 according to the urological procedures performed. In this study, 2 (3.8\%) patients died within the first 30 days, and $5(9.61 \%)$ patients died within the first 3 months. The median age of these patients was 73 (range, 70-88) years. The most common early complication in all patients was urinary leakage in 10 patients, followed by wound infection in six patients. Ureteral anastomotic stenosis that developed in the late period was most common after total cystectomy. Three of these patients developed conduit obstruction caused by benign stricture, and percutaneous nephrostomy was performed. At 2 months after the first operation, one patient developed a fistula between the conduit and the ileum; surgery was performed, and the ileum segment where the fistula developed with the conduit was resected. Then, ureter anastomosis was performed with the Bricker technique. To avoid conduit ischemia, the ileum was anastomosed with the right colon without cecum resection (Table 3).

Evaluation of complications that developed within 30 days postoperatively was made according to Clavien-Dindo classification (8). Complications were then compared as minor complications (grade 0-2) and major complications (grade 3-5). Factors affecting major complications are presented in Table 4.

The shortest and longest follow-up periods of the whole group were 8 and 78 months, respectively, and the mean survival time was 38 months. In terms of survival, no significant difference was found between patients who underwent malignant ureter and benign ureter resections $(p=0.888)$.

\begin{tabular}{|c|c|}
\hline Urological procedure $(n)$ & 52 \\
\hline Partial cystectomy & $20(38.5 \%)$ \\
\hline End to end anstomosis of ureter & $12(23.1 \%)$ \\
\hline Anastomosis to the contralateral ureter & $4(7.7 \%)$ \\
\hline Total cystectomy & $8(15.4)$ \\
\hline Ureteroneocystostomy & $8(15.4)$ \\
\hline \multicolumn{2}{|l|}{ Surgical procedure } \\
\hline Low anterior resection & $22(42.3 \%)$ \\
\hline Left hemicolectomy, anterior resection & $19(36.5 \%)$ \\
\hline Right hemicolectomy & $11(21.1 \%)$ \\
\hline \multicolumn{2}{|l|}{ Ostomy } \\
\hline None & $14(269 \%)$ \\
\hline End colostomy & $20(38.5 \%)$ \\
\hline End ileostomy & $7(13.5 \%)$ \\
\hline Loop ileostomy & $11(21.2 \%)$ \\
\hline \multicolumn{2}{|l|}{ Preoperative double-J catheter } \\
\hline None & $30(57.7 \%)$ \\
\hline Unilateral & $7(13.5 \%)$ \\
\hline Bilateral & $15(28.8 \%)$ \\
\hline \multicolumn{2}{|l|}{ Pathology of the colorectal specimen } \\
\hline Adenocarcinoma & $49(94.2 \%)$ \\
\hline Benign & $2(3.8 \%)$ \\
\hline Gastrointestinal stromal tumor & $1(1.9 \%)$ \\
\hline \multicolumn{2}{|l|}{ Pathology of the urological specimen } \\
\hline Adenocarcinoma & $35(67.3 \%)$ \\
\hline Benign & $16(30.8 \%)$ \\
\hline Gastrointestinal stromal tumor & $1(1.9 \%)$ \\
\hline
\end{tabular}


Özgün et al. Simultaneous Ureter and/or Bladder Reconstructions in Colorectal Malignancies

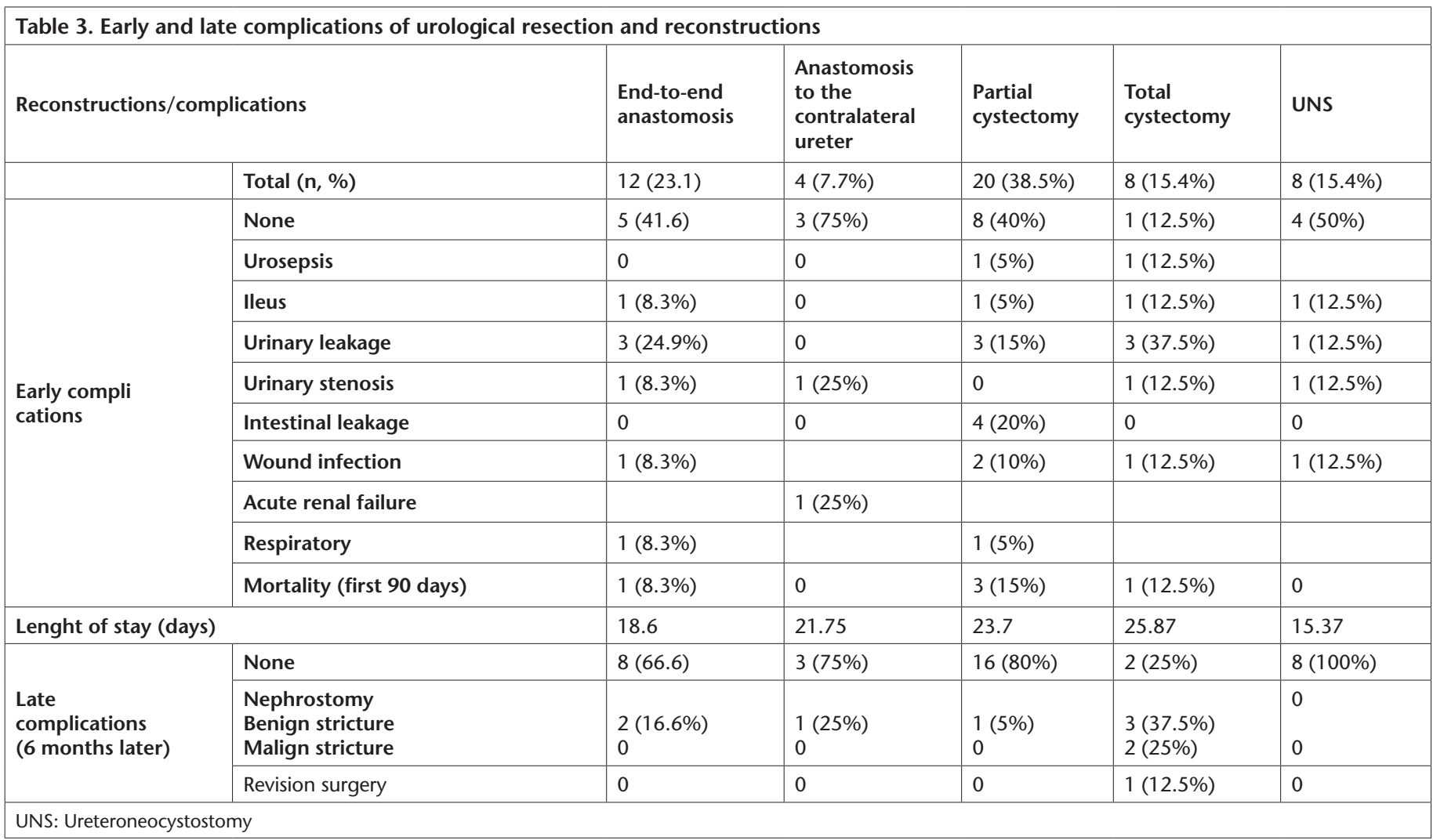

\section{Discussion}

Urological resections and reconstructions performed for $\mathrm{T} 4 \mathrm{~b}$ colorectal malignancies vary, and en bloc resections may provide long-term survival with acceptable mortality and morbidity rates. In our clinic, en bloc resection of urinary organs (ureter and bladder) was performed in 52 (7.9\%) of 657 patients who underwent surgery for colorectal malignancy due to clinical $\mathrm{T} 4 \mathrm{~b}$ tumor. With regard to the early and late outcomes of these patients, the mean survival time was 38 months. The incidence rate of anastomotic stenosis requiring interventional procedures in the late period after these procedures was $17.3 \%$ and the benign stenosis rate was $13.4 \%$.

At the time of diagnosis, $10-20 \%$ of colorectal malignancies are T4 tumors that have invaded the neighboring organs (9). After colorectal surgery, $40 \%$ of the patients develop recurrence, mostly in distant organs. Locoregional recurrence develops within the first 3 years in $10-20 \%$ of the patients (10). The barrier function of the peritoneum against malignant cells is impaired with previous operations, especially after abdominal and pelvic procedures. Recurrent colorectal tumors and peritoneal metastases may be implanted in the retroperitoneum because of the deterioration of the protective barrier function of the peritoneum and the damage caused by lymph node dissections performed in the previous operation, and they may form masses that lead to obstruction, especially in the ureters (11). Longterm survival can be achieved with R0 en bloc resections in tumors with such locoregional recurrence and primarily locally advanced (T4b) tumors (12). In our study, 52 patients with ureter and bladder invasion (clinically $\mathrm{T} 4 \mathrm{~b}$ tumors) underwent en bloc resection. Of these patients, 24 (46.2\%) were operated upon for primary and 28 (53.8\%) for recurrent colorectal malignancy. Despite the numerically higher need for urinary organ resection in recurrent cases, no significant difference was found between the two groups $(p=0.660)$. This numerical difference can be explained by the deterioration of the protective feature of the peritoneum at the first operation and the more frequent retroperitoneal invasion.

In the examination of pathological specimen in en bloc resections, studies have reported that $30-70 \%$ of the patients actually have malignant infiltration, while the remaining patients have inflammation-induced adhesions $(13,14)$. Despite the high rate of benign pathology, en bloc resection is the preferred method because local recurrences increase and survival decreases with the distribution of tumor cells formed by separation of these adhesions $(9,14,15)$. While the 5 -year survival rate is $49-53 \%$ in colorectal malignancies with en bloc resection, attempts to remove the organs separately causes tumor distribution and decreases the survival to $19-21 \%$ even if total resection can be performed (15). In our series, en bloc resection was performed in all patients, and when their primary pathologies were examined, 49 (94.2\%) patients had adenocarcinoma of the colon; moreover, urological specimens were malignant in 35 (67.3\%) and benign in $16(30.8 \%)$ and one patient had GIST. In the present study, the pathological T4b rate was $71.4 \%$. In a study of colorectal malignancies with bladder invasion, Gao et al. (5) stated that the pathological T4b rate was $54 \%$ and the 5 -year survival is not affected by the invasion being malignant 


\begin{tabular}{|c|c|c|c|}
\hline & $\begin{array}{l}\text { Major } \\
\text { Complications (+) }\end{array}$ & $\begin{array}{l}\text { Major } \\
\text { Complications } \\
(-)\end{array}$ & p-value \\
\hline Age (years) & $59.95 \pm 15.74$ & $56.53 \pm 14.05$ & 0.480 \\
\hline \multicolumn{4}{|l|}{ Gender (n) } \\
\hline Male & 18 & 12 & \multirow{2}{*}{0.790} \\
\hline Female & 14 & 8 & \\
\hline \multicolumn{4}{|c|}{ Primary tumor location (n) } \\
\hline Rectum & 14 & 8 & \multirow{3}{*}{0.865} \\
\hline Left colon & 12 & 7 & \\
\hline Right colon & 6 & 5 & \\
\hline Primary tumor & 10 & 14 & \multirow[b]{2}{*}{0.660} \\
\hline Relapse & 10 & 18 & \\
\hline \multicolumn{4}{|l|}{ HIPEC (n) } \\
\hline$(+)$ & 10 & 11 & \multirow{2}{*}{0.089} \\
\hline$(-)$ & 22 & 9 & \\
\hline \multicolumn{4}{|c|}{ Preoperative double-J catheter } \\
\hline$(+)$ & 11 & 11 & \multirow{2}{*}{0.143} \\
\hline$(-)$ & 21 & 9 & \\
\hline \multicolumn{4}{|c|}{ Urological procedures performed } \\
\hline $\begin{array}{l}\text { End to end anastomosis } \\
\text { of ureter }\end{array}$ & 8 & 4 & \multirow{5}{*}{0.554} \\
\hline $\begin{array}{l}\text { Anastomosis to the } \\
\text { contralateral ureter }\end{array}$ & 3 & 1 & \\
\hline $\begin{array}{l}\text { Ureteroneocystostomy } \\
\text { (UNS) }\end{array}$ & 6 & 2 & \\
\hline Partial cystectomy & 12 & 8 & \\
\hline Total cystectomy & 3 & 5 & \\
\hline \multicolumn{4}{|l|}{ Ostomy } \\
\hline$(+)$ & 22 & 16 & \multirow{2}{*}{0.377} \\
\hline$(-)$ & 10 & 4 & \\
\hline
\end{tabular}

or benign. The finding of the present study was similar to that of Gao et al. (5) in that urological organ pathology does not affect survival.

In a large and multicenter study conducted by the PelvEx Collaborative group in patients with locally advanced colorectal cancer, 2.472 patients who underwent pelvic excentration were examined, and the first 30-day major complication rate, which varied by years, was $31.2-45.1 \%$ and the mortality rate as $0 \%$ $7 \%$ (16). In the present study, the first 30-day mortality and major complication rates were $3.9 \%$ and $35.2 \%$, respectively, in accordance with the literature.

Bladder-sparing partial cystectomy can be performed in invasions to the bladder, and the bladder can be primarily repaired. If there is trigon invasion or if the bladder volume will remain too small, en bloc pelvic exentration should be performed (17). No oncological difference was found between total cystectomy or bladder-sparing cystectomy after a negative microscopic surgical margin was performed (17). However, studies have shown that total cystectomy increases complications and impairs quality of life $(18,19)$. In the present study, in accordance with the literature, early urological major complications after total cystectomy were higher than that after partial cystectomy in patients with colorectal cancer, but this difference was not significant (Table 4). Therefore, we can say that bladder-sparing surgery is more advantageous in terms of the postoperative period and quality of life if it is performed without compromising oncological principles. After total cystectomy, urinary continuity can be maintained as continent or noncontinent $(20,21)$. As in our series, noncontinent ileal conduits are preferred more frequently in such cases. We performed orthotropic neobladder reconstructon in one patient, and reconstruction with an ileal conduit in seven patients. In the literature, after ileal conduit, the rates of urinary leakage, ileus, and ileal leakage were $7-27.7 \%$, $22 \%$, and $11.1 \%$, respectively $(20,22)$. As late complications, ureteroenteric anastomosis stenosis and renal failure and abdominal wall-related complications were reported in $7-14 \%$ and $15 \%-65 \%$ of the cases, respectively (20). We performed nephrostomy and dilatation in three patients who underwent ileal conduit in the late period, owing to the development of stenosis for benign reasons. Conduit revision was performed in a patient who developed a fistula between the conduit and ileum 2 months after the first surgery. We performed ureter anastomosis with Bricker technique, and the ileum was anastomosed with the right colon without cecum resection to avoid the conduit ischemia. In two patients, palliative nephrostomy was performed because of recurrence after an average of 13 (6-21) months in the postoperative period, and the Bricker type ileal conduit technique was performed. In a meta-analysis comparing the Bricker and Wallace techniques in terms of late stricture, no difference was found between the two techniques (23). Based on the literature and results of the present study, if the negative margin can be achieved, we think that the early and late outcomes of bladder-sparing surgery will be better; therefore, intraoperative frozen examination is required from the bladder margins.

In the comparison of patients with and without major complications, we found that factors such as age, gender, localization of the primary tumor, requirement of preoperative D], and primary or recurrence of CRC had no effect on major complications. In addition, 21 (40.4\%) patients underwent CRS + HIPEC of the peritoneal metastasis of colorectal cancer. The mean $\mathrm{PCl}$ value of these patients was $6(0-18)$. Six patients with a $\mathrm{PCl}$ value of 0 were those who underwent prophylactic HIPEC. In terms of major complications and mortality, no significant difference was found between patients who underwent HIPEC and those who did not. Moreover, no significant difference was noted in terms of survival between HIPEC and non-HIPEC groups. Studies have shown that the addition of urological procedures increases postoperative complications in patients who underwent CRS + HIPEC, but does not affect long-term survival, so it does not constitute a contraindication for surgery $(24,25)$. Morkavuk et al. (26) found that preoperative hydronephrosis, primary or recurrent tumor, and ureter reconstruction type did not cause a difference in terms of complications in patients who underwent ureter resection simultaneously with CRS + HIPEC (26). We also found that ureter resection and reconstruction types have no 
effect on major complications and mortality. A multicenter study of the BIG-RENAPE group examined ureter resections performed in 7-8\% of patients who underwent CRS + HIPEC and found that reconstruction was better in patients who underwent UNS than in patients who underwent an end-to-end anastomosis (27). We can say that UNS is the type of anastomosis with the least anastomotic stenosis in the late period.

\section{Study Limitations}

This study has some limitations. Although the data of patients who underwent surgery were recorded prospectively, the study was designed retrospectively. The low number of patient groups in the study also makes the study weak. Ureter and bladder resection and reconstruction types constitute a heterogeneous group. By contrast, in the literature regarding such en bloc resections, the numbers are low even in high-volume centers.

\section{Conclusion}

In patients with clinical $\mathrm{T} 4 \mathrm{~b}$ and colorectal malignancies, en bloc resection should be the oncological procedure to be selected, and a surgical plan should be made by urological and colorectal surgeons by making a management plan approved by a multidisciplinary oncology council. In bladder resections, if possible, organ-preserving surgery should be performed with sufficient negative margin. However, it is thought that late outcomes of UNS in ureter reconstruction are better and those of total cystectomy procedure are worse. We recommend performing prospective randomized studies and meta-analyses to reach a definite conclusion.

\section{Acknowledgements}

Publication: The results of the study were not published in full or in part in form of abstracts.

Contribution: There is not any contributors who may not be listed as authors.

Conflict of Interest: No conflict of interest was declared by the authors.

Financial Disclosure: The authors declared that this study received no financial support.

\section{Ethics}

Ethics Committee Approval: The study was planned as a retrospective analysis and before starting the study ethical permission was provided by the local hospital ethics committee. The ethical number of this study is E2-21-355.

Informed Consent: Retrospective study.

Peer-review: Externally and internally peer-reviewed.

\section{Authorship Contributions}

Critical Review: Y.M.Ö., Concept: Y.M.Ö., V.Ö., M.K.Ç., Design: Y.M.Ö., V.Ö., M.K.Ç., O.A., E.B.B., Data Collection or Processing: E.P., O.A., E.T., Analysis or Interpretation: V.Ö., C.C., Literature Search: M.K.Ç., Writing: Y.M.Ö., M.K.Ç.

\section{References}

1. Wasmann KATGM, Klaver CEL, van der Bilt JDW, et al. Subclassification of Multivisceral Resections for T4b Colon Cancer with Relevance for Postoperative Complications and Oncological Risks. J Gastrointest Surg 2020;24:2113-2120.

2. Courtney D, McDermott F, Heeney A, Winter DC. Clinical review: surgical management of locally advanced and recurrent colorectal cancer. Langenbecks Arch Surg 2014;399:33-40.

3. Mukai T, Nagasaki T, Akiyoshi T, et al. Laparoscopic multivisceral resection for locally advanced colon cancer: a single-center analysis of short- and long-term outcomes. Surg Today 2020;50:1024-1031.

4. Nadiradze G, Yurttas C, Königsrainer A, Horvath P. Significance of multivisceral resections in oncologic surgery: A systematic review of the literature. World J Meta-Anal 2019;7:269-289.

5. Gao F, Cao YF, Chen LS, et al. Outcome of surgical management of the bladder in advanced colorectal cancer. Int J Colorectal Dis 2007;22:21-24.

6. Tan GHC, Shannon NB, Chia CS, et al. The impact of urological resection and reconstruction on patients undergoing cytoreductive surgery (CRS) and hyperthermic intraperitoneal chemotherapy (HIPEC). Asian J Urol 2018;5:194-198.

7. Honoré C, Souadka A, Goéré $D$, et al. HIPEC for peritoneal carcinomatosis: does an associated urologic procedure increase morbidity? Ann Surg Oncol 2012;19:104-109.

8. Dindo D, Demartines N, Clavien PA. Classification of surgical complications: a new proposal with evaluation in a cohort of 6336 patients and results of a survey. Ann Surg 2004;240:205-213.

9. Landmann RG, Weiser MR. Surgical management of locally advanced and locally recurrent colon cancer. Clin Colon Rectal Surg 2005; 18:182-189.

10. Larkin JO, O'Connell PR. Multivisceral resection for T4 or recurrent colorectal cancer. Dig Dis 2012;30(Suppl 2):96-101.

11. Sugarbaker PH. Peritoneum as the first-line of defense in carcinomatosis. J Surg Oncol 2007; 95:93-96.

12. Ferenschild FT, Vermaas $M$, Verhoef $C$, et al. Total pelvic exenteration for primary and recurrent malignancies. World J Surg 2009;33:1502-1508.

13. Chen YG, Liu YL, Jiang SX, Wang XS. Adhesion pattern and prognosis studies of T4NOM0 colorectal cancer following en bloc multivisceral resection: evaluation of T4 subclassification. Cell Biochem Biophys 2011;59:1-6.

14. Bartoş A, Bartoş D, Dunca F, et al. Multi-organ resections for colorectal cancer: analysis of potential factors with role in the occurrence of postoperative complications and deaths. Chirurgia 2012; 107:476-482.

15. Lehnert $T$, Methner $M$, Pollok $A$, et al. Multivisceral resection for locally advanced primary colon and rectal cancer: an analysis of prognostic factors in 201 patients. Ann Surg 2002;235:217-225.

16. PelvEx Collaborative. Changing outcomes following pelvic exenteration for locally advanced and recurrent rectal cancer. BJS Open 2019;3516-520.

17. Delacroix SE Jr, Winters JC. Bladder reconstruction and diversion during colorectal surgery. Clin Colon Rectal Surg 2010;23:113-118.

18. Winter DC, Walsh R, Lee G, et al. Local involvement of the urinary bladder in primary colorectal cancer: outcome with en-bloc resection. Ann Surg Oncol 2007;14:69-73.

19. Balbay MD, Slaton JW, Trane N, et al. Rationale for bladder-sparing surgery in patients with locally advanced colorectal carcinoma. Cancer 1999;86:2212-2216.

20. Helewa RM, Park J. Surgery for locally advanced T4 rectal cancer: strategies and techniques. Clin Colon Rectal Surg 2016;29:106-113.

21. Nerli RB, Ghagane SC, Ram P, et al. Bladder invasion in patients with advanced colorectal carcinoma. Indian J Surg Oncol 2018;9:547-551. 
22. Kondo A, Sasaki T, Kitaguchi D, et al. Resection of the urinary bladder for locally advanced colorectal cancer: a retrospective comparison of partial versus total cystectomy. BMC Surg 2019;19:63.

23. Davis NF, Burke JP, McDermott T, et al. Bricker versus Wallace anastomosis: A meta-analysis of ureteroenteric stricture rates after ileal conduit urinary diversion. Can Urol Assoc J 2015;9:E284-90. doi: 10.5489/cuaj.2692.

24. Braam HJ, van Oudheusden TR, de Hingh IH, et al. Urological procedures in patients with peritoneal carcinomatosis of colorectal cancer treated with HIPEC: morbidity and survival analysis. Anticancer Res 2015;35:295-300.

25. Lyon TD, Turner li RM, Nikonow TN, et al. Effect of a concomitant urologic procedure on outcomes following cytoreductive surgery with hyperthermic intraperitoneal chemotherapy. I Surg Oncol 2016;113:218-222.

26. Morkavuk ŞB, Güner $M$, Tez M, Ünal AE. The outcomes of isolated ureteral resection and reconstruction in non-urologic cancer patients who underwent cytoreductive surgery (CRC) and hyperthermic intraperitoneal chemotherapy (HIPEC). World J Surg Oncol 2019; 17:230.

27. Pinar U, Tremblay JF, Passot G, et al; BIG-RENAPE Working Group. Reconstruction after ureteral resection during HIPEC surgery: Reimplantation with uretero-neocystostomy seems safer than end-toend anastomosis. J Visc Surg 2017;154:227-230. 